\title{
Comprehensive Evaluation of Different T-Helper Cell Subsets Differentiation and Function in Rheumatoid Arthritis
}

\author{
Junwei Chen, Junxia Li, Huiying Gao, Caihong Wang, \\ Jing Luo, Zhiqin Lv, and Xiaofeng Li \\ Department of Rheumatology, The Second Hospital of Shanxi Medical University, Shanxi, Taiyuan 030001, China \\ Correspondence should be addressed to Xiaofeng Li, xiaofengli65@gmail.com
}

Received 14 March 2012; Revised 21 May 2012; Accepted 5 June 2012

Academic Editor: Soldano Ferrone

Copyright () 2012 Junwei Chen et al. This is an open access article distributed under the Creative Commons Attribution License, which permits unrestricted use, distribution, and reproduction in any medium, provided the original work is properly cited.

\begin{abstract}
Rheumatoid arthritis (RA) is the most common autoimmune disorder. Loss of Th1/Th2 and Th17/Treg balance has been reported in several inflammatory autoimmune diseases. This study was to investigate Th1, Th2, Th17, and Treg differentiation and related cytokines in RA patients. The frequencies of Th1, Th2, Th17, and Treg cells in peripheral blood of RA patients $(n=76)$ and healthy controls $(n=18)$ were determined by flow cytometry. Eight serum cytokines were analyzed using cytometric bead array. The results demonstrated that RA patients exhibited increased peripheral Th1/Th17 cells and Th1/Th17-related cytokines. However, Th1 cells only reached significant difference at advanced stage, but Th17 at all stages, suggesting more important roles in Th17 cells. For Th2 and Treg cells, there was a different function pattern in RA progression. Although with the increase of DAS28 score, Th2 cell experienced some degree of decrease in RA patients, no significant difference was observed. IL-4 and IL-10 showed a significant increase in RA patients. These indicated that Th2 cells might exert immunosuppression effects mainly by secreting cytokines. Treg cells were found significantly decreased in RA patients, but no difference was observed in TGF- $\beta$ expression, indicating a cell-cell interaction pattern in Treg cell.
\end{abstract}

\section{Introduction}

Rheumatoid arthritis (RA) is the most common chronic, systemic, inflammatory autoimmune disorder, affecting approximately $1 \%$ of the world's population [1]. It is characterized by persistent synovitis, pain, swelling, and progressive destruction of the small joints of the hands and feet, accompanied by functional disability [2], typically presenting between the ages of 30 and 50 years [3]. Thelper (Th) cells have been suggested to play a central role in immune protection. They do so through their capacity to help B cells make antibodies, to induce macrophages to develop enhanced microbicidal activity, to recruit neutrophils, eosinophils, and basophils to sites of infection and inflammation, and, through their production of cytokines and chemokines, to orchestrate the full panoply of immune responses [4-6]. Therefore, Th cells were predicted to exert a crucial role in RA development.
Th cell can differentiate into at least 4 distinct subsets, namely, Th1, Th2, Th17, and Treg cells. These cell subsets can act at different stages of RA disease, at different sites, and with different intensities, to participate in the complex network of cell-cell interactions that govern the development and chronicity of rheumatoid synovitis [7]. Recent studies have indicated that the imbalance of Th1/Th2 and Th17/Treg cells may be responsible for the development and progression of RA [8]. Of them, Th1 and Th17 cells have a proinflammatory role and have been implicated in many inflammatory conditions in humans and mice [9], while an anti-inflammatory role is attributed to Th2 and Treg cells.

Studies also have demonstrated that cytokines are involved in every stage of RA disease through maintaining inflammatory synovitis to promote self-immune and lead to the destruction of the adjacent joint tissue [10]. Distinct Th cell subsets secrete different cytokines and 
these cytokines play different roles in a complex regulatory network. Therefore, the aim of our work was to evaluate Th cell differentiation and corresponding cytokines in patients with RA and different stages of RA.

\section{Materials and Methods}

2.1. Patients and Healthy Controls. Seventy-six patients with active RA, fulfilling the revised classification criteria of the American College of Rheumatology [11], were enrolled in this study. RA activity was estimated according to clinical indices (tender joint and swollen joint count), as well as laboratory markers of inflammation, the erythrocyte sedimentation rate (ESR) and C-reactive protein (CRP), and calculation of the disease activity score (DAS28). Of them, 14 patients showed low disease activity with a DAS28 $\leq 3.2$, 22 had moderate disease activity $(3.2<$ DAS2 $2 \leq 5.1)$, and 40 had high disease activity (DAS28 > 5.1) [12]. Eighteen healthy volunteers were enrolled as controls. Written consent was obtained from all subjects after a full explanation of the procedures.

2.2. Cell Preparation. Approximately, $22 \mathrm{~mL}$ of venous blood was drawn by venipuncture from RA patients $(n=76)$ and healthy controls $(n=18)$. Fifteen milliliters of the $22 \mathrm{~mL}$ sampled was heparinized for the isolation of peripheral blood mononuclear cells (PBMCs), while the remaining $7 \mathrm{~mL}$ was used for the preparation of serum. PBMCs were isolated for the analysis of flow cytometry using FicollHypaque (Lymphoprep, Norway) density gradient centrifugation. The centrifugation was performed at $2000 \mathrm{rpm}$ for $20 \mathrm{~min}$ at $20^{\circ} \mathrm{C}$. Serum was separated from the specimens at $1000 \mathrm{rpm}$ for $15 \mathrm{~min}$ at $20^{\circ} \mathrm{C}$ and stored at $-80^{\circ} \mathrm{C}$ until used for cytokine determination using cytometric bead array (CBA).

2.3. Flow Cytometric Analysis of Th1, Th2, Th17, and Treg Cells. For analysis of Th1, Th2, and Th17 cells, PBMCs were suspended at a final density of $0.5 \times 10^{7}$ cells $/ \mathrm{mL}$ in complete culture medium (RPMI 1640 supplemented with $100 \mathrm{U} / \mathrm{mL}$ penicillin, $100 \mathrm{U} / \mathrm{mL}$ streptomycin, sodium pyruvate, and with $10 \%$ fetal calf serum). Cultures were stimulated for $5 \mathrm{~h}$ with $30 \mathrm{ng} / \mathrm{mL}$ phorbol myristate acetate (PMA), $750 \mathrm{ng} / \mathrm{mL}$ ionomycin, and brefeldin A. Cells were washed in FACS solution and surface-labeled with CD4-APC. Following surface staining, cells were fixed and permeabilized using fixation/permeabilization reagent (Becton Dickinson, USA) and then stained with IFN- $\gamma$-PE (Th1), IL-4-PE (Th2), and IL-17-PE (Th17).

For analysis of Treg cells, PBMCs were aliquoted into tubes without PMA and ionomycin stimulation and surfacelabeled with CD4-FITC and CD25-PE followed by fixation, permeabilization, and intracellular staining with FoxP3FITC.

Labeled cells were washed and analyzed with a FACSCalibur flow cytometer (Becton-Dickinson) using the CellQuest software (Becton-Dickinson). In each case, staining was compared with that of the appropriately labeled isotype control antibody.

2.4. CBA for the Measurement of Cytokine. Analysis of cytokines in serum from RA patients and healthy controls was conducted using a CBA human Th1/Th2/Th17 cytokine kit (Becton Dickinson, USA) and analyzed on a FACSCalibur flow cytometer. Beads were coated with antibodies specifically reacting with each of the cytokines to be detected in the multiplex system. The beads could be differentiated by their sizes and their distinct spectral addresses. Seven cytokines were measured: interleukin (IL)-2 (IL-2), IL-4, IL-6, IL-10, tumor necrosis factor (TNF- $\alpha$ ), interferon- $\gamma$ (IFN- $\gamma$ ), IL$17 \mathrm{~A}$, and transforming growth factor- $\beta$ (TGF- $\beta$ ). Standard curves were determined for each cytokine from a range of $1-5000 \mathrm{pg} / \mathrm{mL}$. Quantity ( $\mathrm{pg} / \mathrm{mL}$ ) of respective cytokine was calculated using CBA software.

2.5. Statistical Analysis. The changes of Th1, Th2, Th17, and Treg cells between RA and control groups were measured by mean \pm standard deviation and difference was performed using $t$-test. That $P<0.05$ was considered as statistically significant. However, the data resulting from cytokine measurement were expressed as median \pm interquartile range. The differences between RA and control groups were assessed by Wilcoxon test, but the differences between different DAS28 RA groups were determined by Kruskal-Wallis $H$ test. $P$-values less than 0.001 were considered significant.

\section{Results}

3.1. The Frequencies of Th1, Th2, Th17, and Treg Cells in Peripheral Blood of RA Patients. The frequencies of Th1, Th2, Th17, and Treg cells in peripheral blood of RA patients with low, moderate, and high DAS28 scores, and healthy controls were determined by flow cytometry. Overall, there was a significant increase in Th1 $(t=2.251, P=0.028)$ and Th17 cells $(t=2.266, P=0.026)$ of RA patients when compared with healthy controls, while significant decrease was present in Treg cells of RA patients in comparison with healthy controls $(t=2.316, P=0.023)$ (Table 1$)$. Although Th2 cells showed a little increase in RA patients, no significant difference was observed ( $t=0.349, P=0.728$ ).

Further, the frequency of Th1 cells was only significant higher in RA patients with high DAS28 score $(t=2.623$, $P=0.012)$ than those in healthy controls, but not in low $(t=1.583, P=0.134)$ and moderate DAS28 score $(t=1.750, P=0.091)$. However, Th17 cells were shown a significant increase in all RA stages $(t=2.120, P=0.047$ for low DAS28 score; $t=2.391, P=0.023$ for moderate DAS28 score; $t=2.449, P=0.018$ for high DAS28 score), indicating more important roles of Th17 in RA development. Identically, Treg cells exhibited a significant decrease only in RA patients with high DAS28 score $(t=2.429, P=0.019)$, not in low $(t=0.156, P=0.878)$ and moderate DAS28 score $(t=1.554, P=0.129)$. Although with the increase of DAS28 score, Th2 cells experienced some degree of decrease in RA patients, no significant difference was observed $(t=0.464$, 
TABLE 1: The frequencies of Th1, Th2, Th17, and Treg cell in RA patients and control.

\begin{tabular}{lccccc}
\hline Group & $n$ & Th1 cell & Th2 cell & Th17 cell & Treg cell \\
\hline RA patients & 76 & $6.2 \pm 4.5^{*}$ & $1.7 \pm 0.9$ & $1.1 \pm 0.9^{*}$ & $1.8 \pm 1.2^{*}$ \\
DAS28 $\leq 3.2$ & 14 & $5.1 \pm 3.9$ & $1.8 \pm 0.4$ & $0.9 \pm 0.4^{*}$ & $2.5 \pm 0.6$ \\
$3.2<$ DAS28 $\leq 5.1$ & 22 & $5.6 \pm 4.7$ & $1.7 \pm 0.4$ & $1.0 \pm 0.6^{*}$ & $1.8 \pm 1.45$ \\
DAS28 $>5.1$ & 40 & $6.8 \pm 4.6^{*}$ & $1.7 \pm 1.0$ & $1.3 \pm 1.0^{*}$ & $1.6 \pm 1.2^{*}$ \\
Control & 18 & $2.9 \pm 1.6$ & $1.6 \pm 1.0$ & $0.6 \pm 0.4$ & $2.6 \pm 1.8$ \\
\hline
\end{tabular}

Values are expressed as mean \pm standard deviation or number; $t$-test was used for statistical analysis; ${ }^{*} P<0.05$ was considered as statistically significant.

TABLE 2: Th cell-related cytokine changes of RA patients compared with healthy control.

\begin{tabular}{|c|c|c|c|c|}
\hline Cytokine & $\mathrm{RA}(n=76)$ & Control $(n=18)$ & $Z$ & $P$ \\
\hline TGF- $\beta$ & $159.9 \pm 13.2$ & $156.0 \pm 6.4$ & 1.8 & 0.078 \\
\hline IL-17A & $48.7 \pm 20.2^{*}$ & $32.5 \pm 7.1$ & 4.2 & $<0.001$ \\
\hline IFN- $\gamma$ & $5.4 \pm 3.2^{*}$ & $3.6 \pm 0.7$ & 3.4 & 0.001 \\
\hline TNF- $\alpha$ & $4.9 \pm 4.3^{*}$ & $3.5 \pm 0.8$ & 3.7 & $<0.001$ \\
\hline IL-10 & $7.1 \pm 3.4^{*}$ & $5.1 \pm 2.3$ & 4.0 & $<0.001$ \\
\hline IL-6 & $15.8 \pm 42.1^{*}$ & $6.8 \pm 3.2$ & 4.3 & $<0.001$ \\
\hline IL-4 & $14.6 \pm 5.0^{*}$ & $10.9 \pm 2.0$ & 4.3 & $<0.001$ \\
\hline IL-2 & $14.4 \pm 4.6^{*}$ & $11.1 \pm 1.4$ & 4.7 & $<0.001$ \\
\hline
\end{tabular}

Values are expressed as median \pm interquartile range; the differences between RA and control groups were assessed by Wilcoxon test; ${ }^{*} P$ values less than 0.001 were considered significant.

$P=0.647$ for low DAS28 score; $t=0.263, P=0.794$ for moderate DAS28 score; $t=0.753, P=0.455$ for high DAS28 score).

3.2. Th1, Th2, Th17, and Treg-Related Cytokines in the Serum from RA Patients. Eight Th cell-related cytokines in serum of RA patients and healthy controls were measured using flow cytometric bead-based technology. Compared with traditional ELISA method, this new technology allows for evaluation of multiple cytokines in a single sample and utilization of minimal sample volumes to obtain data [13]. As shown in Table 2, RA group exhibited higher levels of serum IL-2, IL-4, IL-6, IL-10, TNF- $\alpha$, IFN- $\gamma$, and IL-17A than those of healthy controls (all $P<0.001$ ) (Table 2). Further, the detailed cytokines changes were analyzed along with RA progression (Table 3 ). Th1 cells mainly secrete IL- 2, TNF- $\alpha$, and IFN- $\gamma$. In contrast to healthy control, IL-2 showed significant increase in RA patients with low, moderate, and high DAS28 scores $(P<0.001)$, yet, IFN$\gamma$ only significantly increased in RA patients with high DAS28 scores $(P<0.001)$, and TNF- $\alpha$ was significantly increased in RA patients with low and high DAS28 score $(P<0.001)$. IL-4 and IL-10 mainly result from Th2 cells. IL-4 was significantly enhanced in RA patients with low and high DAS28 scores $(P<0.001)$, but IL-10 was significantly enhanced in RA patients with moderate and high DAS28 scores $(P<0.001)$. IL-17A and IL-6 are produced from Th17 cells, which all showed a significant increase in RA patients with moderate and high DAS28 scores $(P<0.001)$. Remarkably, no significant increase was observed in TGF- $\beta$ expression of RA patients compared with healthy control, which commonly result from Treg cells.

\section{Discussions}

4.1. Th1/Th2 Balance. Commonly, abnormal activation of Th1 cells is suggested as the critical event in most organspecific autoimmune diseases, while Th2 cells are responsible for allergic inflammatory diseases and asthma [14]. Animal experiments have also demonstrated that RA is a Th1-celldriven chronic inflammatory disorder [15]. In the meanwhile of Th1-mediated inflammation, Th1 also can prevent $\mathrm{CD}^{+}{ }^{+} \mathrm{T}$ cells from differentiating into Th2 cells, and even interfere anti-inflammatory cytokines produced from Th2, such as IL-4. Thus, excessive production of inflammatory cytokines,but deficient anti-inflammatory mediators will finally lead to Th1/Th2 imbalance.

In this study, we firstly investigated the frequencies of Th1 and Th2 cells in the peripheral blood of patients with RA. The results demonstrated that the frequency of Th1 cells was significantly higher in RA patients than those in healthy controls. Although with the increase of DAS28 score, Th2 cells experienced some degree of decrease in RA patients, no significant difference was observed. One possible explanation is a competitive inhibition to Th1 differentiation, which also seemed to be in accordance with our result that significant difference of Th1 cell was only observed in RA patients with high DAS28 score, but not in low and moderate DAS28 scores.

Further serum cytokine profile was investigated in patients with RA. The principal inflammatory cytokines produced from Th1 were IFN- $\gamma$, IL- 2 , and TNF- $\alpha$, which 
TABLE 3: Th cell-related cytokine changes of RA patients with different DAS28 scores.

\begin{tabular}{lcccccc}
\hline Cytokine & Low DAS28 $(n=14)$ & Moderate DAS28 $(n=22)$ & High DAS28 $(n=40)$ & Control $(n=18)$ & $H$ \\
\hline TGF- $\beta$ & $158.6 \pm 6.4$ & $157.4 \pm 13.0$ & $159.8 \pm 11.9$ & $156.0 \pm 6.4$ & 2.9 & 0.404 \\
IL-17A & $36.4 \pm 17.8$ & $44.0 \pm 12.7^{*}$ & $51.4 \pm 23.1^{*}$ & $32.5 \pm 7.1$ & 19.6 & $<0.001$ \\
IFN- $\gamma$ & $3.3 \pm 0.4$ & $4.7 \pm 2.2$ & $5.7 \pm 3.7^{*}$ & $3.6 \pm 0.7$ & 16.4 & 0.001 \\
TNF- $\alpha$ & $4.8 \pm 2.9^{*}$ & $4.1 \pm 3.1$ & $4.9 \pm 5.6^{*}$ & $3.5 \pm 0.8$ & 16.6 & 0.001 \\
IL-10 & $5.9 \pm 2.0$ & $6.8 \pm 4.3^{*}$ & $7.4 \pm 4.3^{*}$ & $5.1 \pm 2.3$ & 17.9 & $<0.001$ \\
IL-6 & $9.5 \pm 53.5$ & $29.7 \pm 57.4^{*}$ & $12.8 \pm 43.4^{*}$ & $6.8 \pm 3.2$ & 27.8 & $<0.001$ \\
IL-4 & $13.1 \pm 2.0^{*}$ & $13.8 \pm 6.9$ & $14.8 \pm 4.8^{*}$ & $10.9 \pm 2.0$ & 20.3 & $<0.001$ \\
IL-2 & $12.5 \pm 2.5^{*}$ & $13.0 \pm 4.6^{*}$ & $14.3 \pm 5.1^{*}$ & $11.1 \pm 1.4$ & 23.8 & $<0.001$ \\
\hline
\end{tabular}

Values are expressed as median \pm interquartile range; the differences between different DAS28 RA groups were determined by Kruskal-Wallis $H$ test; ${ }^{*} P$ values less than 0.001 were considered significant.

all have been demonstrated involved in RA development. For example, IFN- $\gamma$ is important in activating macrophages to increase their microbicidal activity [16] and improve the activity of natural killer (NK) cells to stimulate RA inflammatory reaction $[17,18]$. IL-2 production is important for CD4 T-cell memory [19]. TNF- $\alpha$ can induce intercellular adhesion molecule (ICAM) expression in endothelial cells (such as ICAM-1), promote adhesion and penetration of leukocyte and vascular endothelium, and finally lead to local inflammation. In addition, TNF- $\alpha$ also can promote synovial cells, macrophages, fibroblast, and cartilage cells to produce IL-1, IL- 8 , and TNF- $\alpha$ itself and aggravate tissue damage [20]. As expected, RA group exhibited higher levels of serum IL-2, TNF- $\alpha$, and IFN- $\gamma$ than those of healthy controls. In contrast with healthy control, IL-2 showed a significant increase in RA patients at all stages. Yet, TNF- $\alpha$ was firstly significantly increased at acute sage (low DAS28 score) and then at the advanced stage (high DAS28 score). IFN- $\gamma$ was only significantly increased in RA patients with high DAS28 score. These findings support the idea that IL2 is a dominant factor in driving Th1 differentiation. IL-4 and IL-10 are mainly produced from Th2 cells. IL-4 plays a positive feedback regulation role in Th2 differentiation $[21,22]$. However, IL-10 inhibits Th1 cells proliferation [23]. IL-4 and IL-10 also showed a higher level in RA patients in our study. IL-4 was significantly enhanced in RA patients with low and high DAS28 scores $(P<0.001)$, but IL-10 was significantly enhanced in RA patients with moderate and high DAS28 scores $(P<0.001)$.

Overall, these results suggested that TNF- $\alpha$, IFN- $\gamma$, IL- 2 IL-4, and IL-10 all involved in RA progression. IFN- $\gamma$, IL-2, and TNF- $\alpha$ contributed to the polarization toward Th1 cells and reached the maximum at the advanced stage of RA. Upregulated expression of IL-4 and IL-10 showed resistance to these processes. More remarkably, there was no significant increase in Th2 cells as mentioned above, raising a possibility that Th2 cells may play its immune regulation role mainly through producing cytokines, but not itself.

4.2. Th17/Treg Balance. Th1/Th2 imbalance can partially explain the pathogenesis of RA, however, there are still a number of incontrovertible facts, such as the virtual absence of IFN- $\gamma$ within the rheumatoid synovium, the lack of efficacy in most patients of monoclonal antibodies to IFN$\gamma$, and the paradoxical effects of IFN- $\gamma$, which alleviates established arthritis in mice but promotes arthritis flares in healthy mice [8]. The identification of Treg cells and Th17 cells has opened up a vast field of research into the potential roles of these cells in controlling RA disease.

Our result showed that Th17 cells significantly increased in RA patients at all stages compared with healthy control, which was in accordance with previous reports [24]. In addition to stimulating synovioblast, macrophage, and other lymphocyte to secrete inflammatory mediators, recent studies [25] have suggested that Th17 cells can also induce RANKL upregulated expression especially in the advanced stage, which is a necessary requisite for the activation of osteoclast and bone absorption [26]. Although the immunoregulation mechanism is not well known, Treg cells are suggested to exert an influence on immune inhibition to antagonize Th17 cells differentiation. As expected, Treg cells were found a significant decrease in RA patients compared with healthy control.

Identically, Th17 and Treg-related cytokine were also investigated in patients with RA. Th17 cells mainly produce IL-17A, IL-6, and TNF- $\alpha$ cytokine. IL-17A plays an important role in inducing inflammation by mediating the generation of other cytokine and chemokine $[27,28]$. TNF$\alpha$ and IL- 6 are also another crucial inflammation mediators. However, different mechanisms are present in TNF- $\alpha$ and IL6 associated with RA. IL- 6 seems not to directly stimulate synovial fibroblast and chondrocyte to synthetize PGE2 and collagenase, but only improve the efficacy of TNF- $\alpha$ in RA [29]. Consistent with previous reports, our results also indicated that RA group exhibited higher levels of serum IL6 , TNF- $\alpha$, and IL-17A than those of healthy controls. Also IL-17A and IL-6 showed a significant increase in RA patients with moderate and high DAS28 scores $(P<0.001)$, but not in low DAS28 score, demonstrating that they participated in RA progression. TGF- $\beta$ is a major cytokine produced from Treg cells. TGF- $\beta$ can induce inactive $\mathrm{CD} 4^{+}$Th cells to differentiate into Treg cells. Nevertheless, Treg cells were found significantly decreased in RA patients at advanced stage, indicating that Treg cells may play an important role in immunosuppression through cell-cell interaction, not cytokine $[30,31]$. 
Recent researches suggest that TGF- $\beta$ is also essential for the generation of Th17 cells [32]. Therefore, TGF$\beta$ was predicted to participate in both Th17 and Treg cells differentiation to maintain the balance between Th17 and Treg cells at early stage of RA [33, 34]. However, at advanced stage, IL- 6 further stimulated Th17 differentiation, but inhibited Treg differentiation, which was demonstrated that Treg development would be enhanced when IL-6 was blocked [32, 35]. Ultimately, Th17/Treg imbalance occurred.

\section{Conclusions}

The imbalance of Th1/Th2 and Th17/Treg cells is proposed responsible for the development and progression of RA. All kinds of cytokines produced from different Th cells are not present independently, but to form interaction network. Our results have demonstrated that Th1 cells only reach significant difference at advanced stage, but Th17 at all stages, indicating more important roles in Th17 cells [36]. Although Th2 and Treg cells both exert anti-inflammatory function, there is a different pattern in RA progression, that is, Th2 cells by secreting cytokines, on the contrary, cell-cell interaction pattern in Treg cells. Nevertheless, further studies are needed to confirm our conclusion.

\section{Conflict of Interests}

All authors including J. Chen, J. Li, H. Gao, C. Wang, G. Zhang, H. Wen, J. Luo, Z. Lv, and X. Li declared that they have no conflict of interests to state.

\section{References}

[1] S. Raychaudhuri, E. F. Remmers, A. T. Lee et al., "Common variants at CD40 and other loci confer risk of rheumatoid arthritis," Nature Genetics, vol. 40, no. 10, pp. 1216-1223, 2008.

[2] K. Nishimura, D. Sugiyama, Y. Kogata et al., "Meta-analysis: diagnostic accuracy of anti-cyclic citrullinated peptide antibody and rheumatoid factor for rheumatoid arthritis," Annals of Internal Medicine, vol. 146, no. 11, pp. 797-808, 2007.

[3] V. Majithia and S. A. Geraci, "Rheumatoid arthritis: diagnosis and management," American Journal of Medicine, vol. 120, no. 11, pp. 936-939, 2007.

[4] J. Zhu and W. E. Paul, "CD4 T cells: fates, functions, and faults,” Blood, vol. 112, no. 5, pp. 1557-1569, 2008.

[5] D. Gómez-Martín, M. Díaz-Zamudio, J. Romo-Tena, M. J. Ibarra-Sánchez, and J. Alcocer-Varela, "Follicular helper $\mathrm{T}$ cells poise immune responses to the development of autoimmune pathology," Autoimmunity Reviews, vol. 10, no. 6, pp. 325-330, 2011.

[6] W. Dong, P. Zhu, Y. Wang, and Z. Wang, "Follicular helper T cells in systemic lupus erythematosus: a potential therapeutic target," Autoimmunity Reviews, vol. 10, no. 6, pp. 299-304, 2011.

[7] W. Wang, S. Shao, Z. Jiao, M. Guo, H. Xu, and S. Wang, "The Th17/Treg imbalance and cytokine environment in peripheral blood of patients with rheumatoid arthritis," Rheumatology International, vol. 32, no. 4, pp. 887-893, 2011.
[8] M. C. Boissier, E. Assier, G. Falgarone, and N. Bessis, "Shifting the imbalance from Th1/Th2 to Th17/treg: the changing rheumatoid arthritis paradigm," Joint Bone Spine, vol. 75, no. 4, pp. 373-375, 2008.

[9] V. Dardalhon, T. Korn, V. K. Kuchroo, and A. C. Anderson, "Role of Th1 and Th17 cells in organ-specific autoimmunity," Journal of Autoimmunity, vol. 31, no. 3, pp. 252-256, 2008.

[10] F. M. Brennan and I. B. McInnes, "Evidence that cytokines play a role in rheumatoid arthritis," Journal of Clinical Investigation, vol. 118, no. 11, pp. 3537-3545, 2008.

[11] F. C. Arnett, S. M. Edworthy, D. A. Bloch et al., "The American Rheumatism Association 1987 revised criteria for the classification of rheumatoid arthritis," Arthritis and Rheumatism, vol. 31, no. 3, pp. 315-324, 1988.

[12] V. P. K. Nell, K. P. Machold, G. Eberl, T. A. Stamm, M. Uffmann, and J. S. Smolen, "Benefit of very early referral and very early therapy with disease-modifying anti-rheumatic drugs in patients with early rheumatoid arthritis," Rheumatology, vol. 43, no. 7, pp. 906-914, 2004.

[13] E. Morgan, R. Varro, H. Sepulveda et al., "Cytometric bead array: a multiplexed assay platform with applications in various areas of biology," Clinical Immunology, vol. 110, no. 3, pp. 252-266, 2004.

[14] K. M. Murphy and S. L. Reiner, "The lineage decisions of helper T cells," Nature Reviews Immunology, vol. 2, no. 12, pp. 933-944, 2002.

[15] H. E. Barksby, S. R. Lea, P. M. Preshaw, and J. J. Taylor, "The expanding family of interleukin-1 cytokines and their role in destructive inflammatory disorders," Clinical and Experimental Immunology, vol. 149, no. 2, pp. 217-225, 2007.

[16] D. T. T. Hang, J. Y. Song, M. Y. Kim, J. W. Park, and Y. K. Shin, "Involvement of NF- $\kappa \mathrm{B}$ in changes of IFN- $\gamma$-induced CIITA/MHC-II and iNOS expression by influenza virus in macrophages," Molecular Immunology, vol. 48, no. 9-10, pp. 1253-1262, 2011.

[17] G. Palmer, F. Mezin, C. E. Juge-Aubry, C. Plater-Zyberk, C. Gabay, and P. A. Guerne, "Interferon $\beta$ stimulates interleukin 1 receptor antagonist production in human articular chondrocytes and synovial fibroblasts," Annals of the Rheumatic Diseases, vol. 63, no. 1, pp. 43-49, 2004.

[18] O. Aravena, B. Pesce, L. Soto et al., "Anti-TNF therapy in patients with rheumatoid arthritis decreases Th1 and Th17 cell populations and expands IFN-[gamma]-producing NK cell and regulatory $\mathrm{T}$ cell subsets," Immunobiology, vol. 216, no. 12, pp. 1256-1263, 2011.

[19] A. Manzo, S. Paoletti, M. Carulli et al., "Systematic microanatomical analysis of CXCL13 and CCL21 in situ production and progressive lymphoid organization in rheumatoid synovitis," European Journal of Immunology, vol. 35, no. 5, pp. 1347-1359, 2005.

[20] J. M. Dayer, "Interleukin 1 or tumor necrosis factor- $\alpha$ : which is the real target in rheumatoid arthritis?" Journal of Rheumatology, vol. 29, no. 65, pp. 10-15, 2002.

[21] B. Manoury-Schwartz, G. Chiocchia, N. Bessis et al., "High susceptibility to collagen-induced arthritis in mice lacking IFN- $\gamma$ receptors," Journal of Immunology, vol. 158, no. 11, pp. 5501-5506, 1997.

[22] K. Vermeire, H. Heremans, M. Vandeputte, S. Huang, A. Billiau, and P. Matthys, "Accelerated collagen-induced arthritis in IFN- $\gamma$ receptor-deficient mice," Journal of Immunolog $y$, vol. 158, no. 11, pp. 5507-5513, 1997.

[23] F. Brentano, D. Kyburz, O. Schorr, R. Gay, and S. Gay, "The role of Toll-like receptor signalling in the pathogenesis of arthritis," Cellular Immunology, vol. 233, no. 2, pp. 90-96, 2005. 
[24] M. Chabaud, J. M. Durand, N. Buchs et al., "Human interleukin-17: AT cell-derived proinflammatory cytokine produced by the rheumatoid synovium," Arthritis \& Rheumatism, vol. 42, no. 5, pp. 963-970, 1999.

[25] K. Raza, D. Scheel-Toellner, C. Y. Lee et al., "Synovial fluid leukocyte apoptosis is inhibited in patients with very early rheumatoid arthritis," Arthritis Research and Therapy, vol. 8, no. 4, article R120, 2006.

[26] F. Fossiez, O. Djossou, P. Chomarat et al., "T cell interleukin17 induces stromal cells to produce proinflammatory and hematopoietic cytokines," Journal of Experimental Medicine, vol. 183, no. 6, pp. 2593-2603, 1996.

[27] M. Feldmann, F. M. Brennan, and R. N. Maini, "Role of cytokines in rheumatoid arthritis," Annual Review of Immunology, vol. 14, pp. 397-440, 1996.

[28] N. Y. A. Hemdan, G. Birkenmeier, G. Wichmann et al., "Interleukin-17-producing T helper cells in autoimmunity," Autoimmunity Reviews, vol. 9, no. 11, pp. 785-792, 2010.

[29] H. Nakahara, J. Song, M. Sugimoto et al., "Anti-interleukin6 receptor antibody therapy reduces vascular endothelial growth factor production in rheumatoid arthritis," Arthritis and Rheumatism, vol. 48, no. 6, pp. 1521-1529, 2003.

[30] N. Nishimoto, K. Yoshizaki, N. Miyasaka et al., "Treatment of rheumatoid arthritis with humanized anti-interleukin6 receptor antibody: a multicenter, double-blind, placebocontrolled trial," Arthritis and Rheumatism, vol. 50, no. 6, pp. 1761-1769, 2004.

[31] E. M. Shevach, "From vanilla to 28 flavors: multiple varieties of T regulatory cells," Immunity, vol. 25, no. 2, pp. 195-201, 2006.

[32] P. J. Cejas, M. C. Walsh, E. L. Pearce et al., "TRAF6 inhibits Th17 differentiation and TGF- $\beta$-mediated suppression of IL2," Blood, vol. 115, no. 23, pp. 4750-4757, 2010.

[33] C. L. Langrish, Y. Chen, W. M. Blumenschein et al., "IL-23 drives a pathogenic $\mathrm{T}$ cell population that induces autoimmune inflammation," Journal of Experimental Medicine, vol. 201, no. 2, pp. 233-240, 2005.

[34] M. Veldhoen, R. J. Hocking, C. J. Atkins, R. M. Locksley, and B. Stockinger, "TGF $\beta$ in the context of an inflammatory cytokine milieu supports de novo differentiation of IL-17-producing $\mathrm{T}$ cells," Immunity, vol. 24, no. 2, pp. 179-189, 2006.

[35] P. R. Mangan, L. E. Harrington, D. B. O'Quinn et al., "Transforming growth factor- $\beta$ induces development of the T H17 lineage," Nature, vol. 441, no. 7090, pp. 231-234, 2006.

[36] J. P. Van Hamburg, P. S. Asmawidjaja, N. Davelaar et al., “Th17 cells, but not Th1 cells, from patients with early rheumatoid arthritis are potent inducers of matrix metalloproteinases and proinflammatory cytokines upon synovial fibroblast interaction, including autocrine interleukin-17A production," Arthritis and Rheumatism, vol. 63, no. 1, pp. 73-83, 2011. 


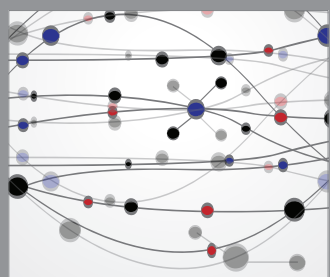

The Scientific World Journal
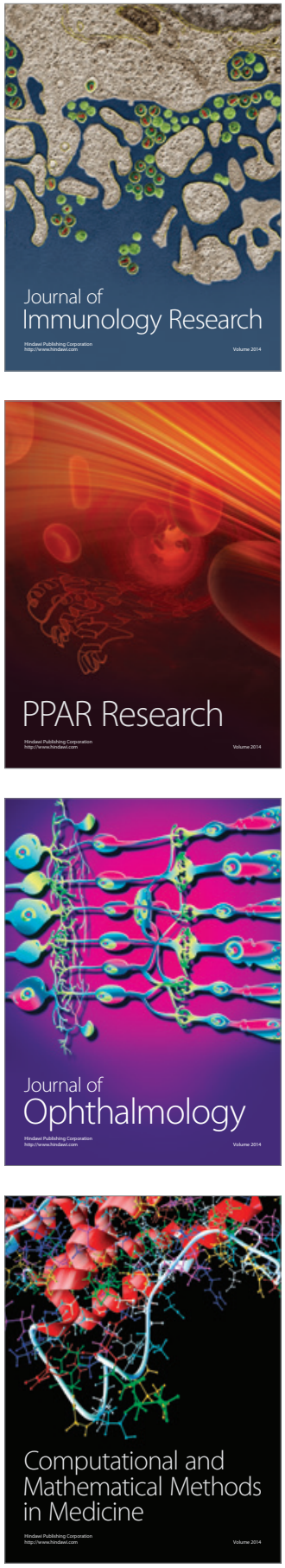

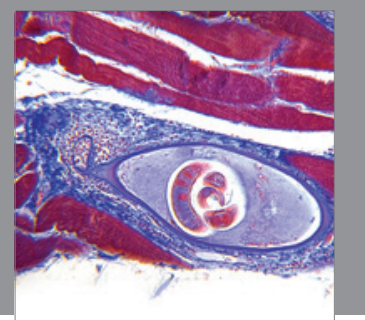

Gastroenterology

Research and Practice
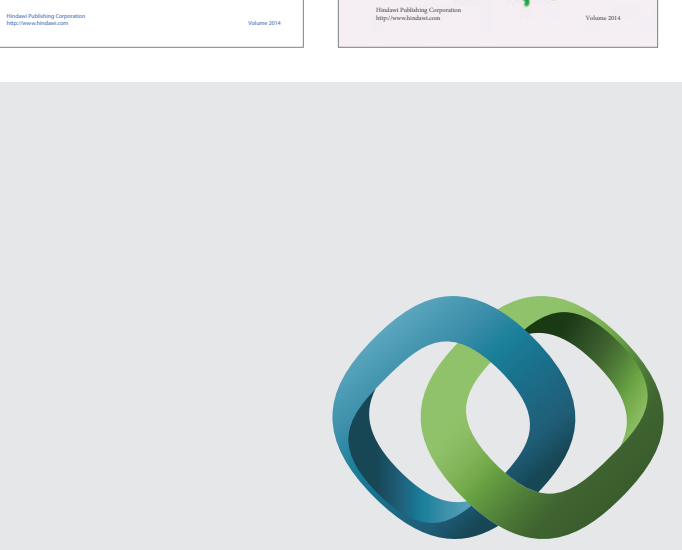

\section{Hindawi}

Submit your manuscripts at

http://www.hindawi.com
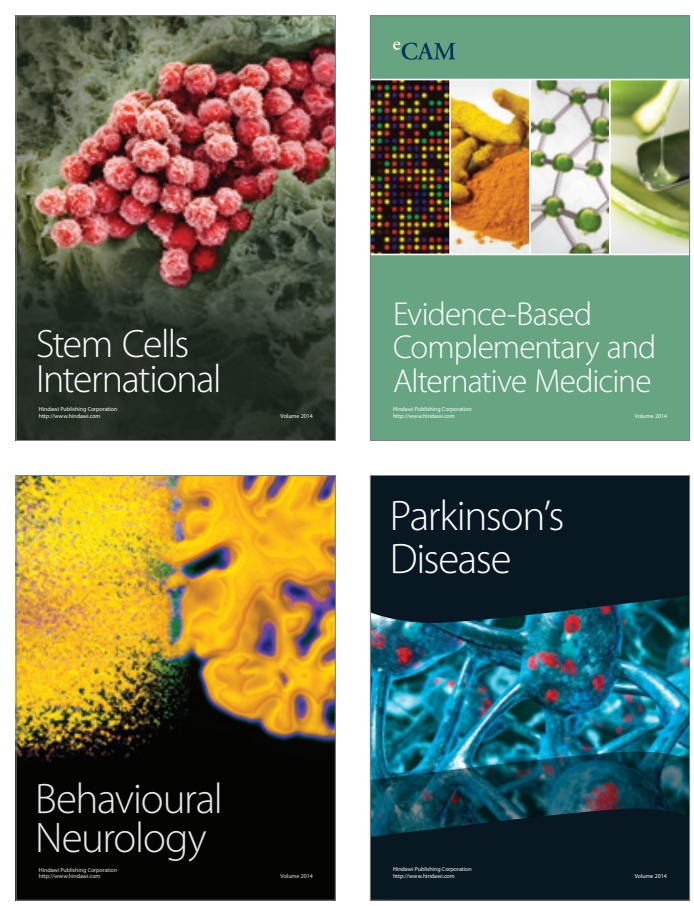

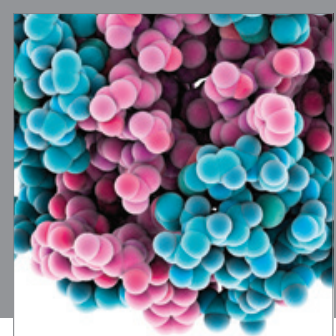

Journal of
Diabetes Research

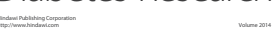

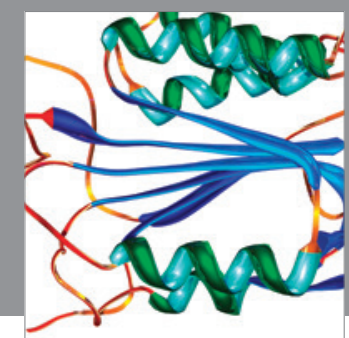

Disease Markers
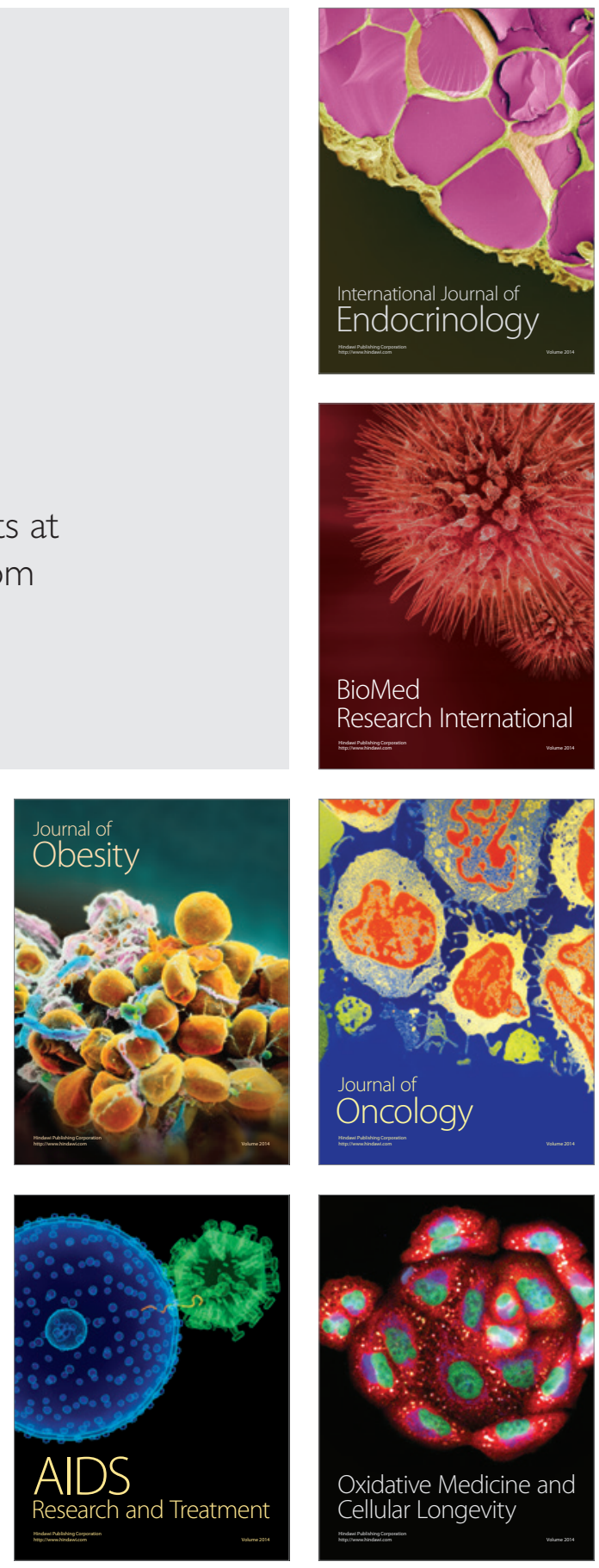